\title{
THE ROW RANK OF A SUBRING OF A MATRIX RING
}

\author{
M. S. LI AND J. M. ZELMANOWITZ
}

\begin{abstract}
For $R$ a subring of an $n \times n$ matrix ring $M_{n}(\Delta)$ over a division ring $\Delta$, we examine an invariant called the row rank of $R$. Roughly speaking, the row rank of $R$ is the largest integer $k$ such that $R$ contains all $k$-rowed matrices over a left order in $\Delta$. The row rank of $R$ is then an integer between 0 and $n$; and we will see that row $\operatorname{rank} R \geq 1$ means that $M_{n}(\Delta)$ is the maximal left quotient $\operatorname{ring}$ of $R$, while row $\operatorname{rank} R=n$ signifies that $M_{n}(\Delta)$ is the classical left quotient ring of $R$. Thus row rank provides a link between maximal and classical quotient rings for rings of this type. A description of the subrings $R$ with row rank $R \geq k$ is obtained which subsumes and generalizes earlier theorems of Faith-Utumi and Zelmanowitz, respectively, for the cases row $\operatorname{rank} R=n$ and row $\operatorname{rank} R \geq 1$.
\end{abstract}

Introduction. Here a subring $R$ of a ring $S$ will be called a large subring of $S$ if for every $0 \neq t \in S$ there exists $r \in R$ with $0 \neq r t \in R$. We will be concerned with the situation when $S=M_{n}(\Delta)$, the ring of $n \times n$ matrices over a division ring $\Delta$. For such subrings the following result was obtained in [4].

THEOREM 1. The following conditions are equivalent for a subring $R$ of $M_{n}(\Delta)$, $\Delta$ a division ring.

(1.i) $R$ is a large subring of $M_{n}(\Delta)$.

(1.ii) There exist matrix units $\left\{e_{i j} \mid 1 \leq i, j \leq n\right\}$ for $M_{n}(\Delta)$ and a left order $D$ in $\Gamma=\left\{s \in M_{n}(\Delta) \mid s e_{i j}=e_{i j} s, 1 \leq i, j \leq n\right\}$ such that

$$
R \supseteq D e_{11}+D e_{12}+\cdots+D e_{1 n} \cong\left(\begin{array}{cccc}
D & D & \ldots & D \\
0 & 0 & \ldots & 0 \\
\vdots & \vdots & & \vdots \\
0 & 0 & \ldots & 0
\end{array}\right) \text {. }
$$

Recall that $\left\{e_{i j} \mid 1 \leq i, j \leq n\right\}$ is a set of matrix units for $M_{n}(\Delta)$ if and only if $\sum_{i=1}^{n} e_{i i}=1$ and $e_{i j} e_{k l}=\delta_{j k} e_{i l}$ for every choice of $i, j, k, l$; then the centralizer $\Gamma=\left\{s \in M_{n}(\Delta) \mid s e_{i j}=e_{i j} s, 1 \leq i, j \leq n\right\}$ is a division ring isomorphic to $\Delta[\mathbf{3}$, p. 52]. Condition (1.i) says precisely that $M_{n}(\Delta)$ is the maximal left quotient ring of $R[\mathbf{1}, \mathrm{p} .66]$.

The possibility of the preceding theorem was first raised as a question by C. Faith [1, Open Problem 13], and was prompted by the following well-known theorem of Faith and Utumi [2].

THEOREM $n$. The following conditions are equivalent for a subring $R$ of $M_{n}(\Delta)$ $\Delta$ a division ring.

Received by the editors December 27, 1985.

1980 Mathematics Subject Classification (1985 Revision). Primary 15A30, 16A42, 16A48; Secondary $15 \mathrm{~A} 33,16 \mathrm{~A} 18,16 \mathrm{~A} 40,16 \mathrm{~A} 64$. 
(n.i) Given any $t \in M_{n}(\Delta)$ there exists $r \in R$ with $r$ invertible in $M_{n}(\Delta)$ and with $r t \in R$.

(n.ii) There exist matrix units $\left\{e_{i j} \mid 1 \leq i, j \leq n\right\}$ for $M_{n}(\Delta)$ and a left order $D$ in $\Gamma=\left\{s \in M_{n}(\Delta) \mid s e_{i j}=e_{i j} s, 1 \leq i, j \leq n\right\}$ such that $R \supseteq \sum_{1 \leq i, j \leq n} D e_{i j} \cong M_{n}(D)$.

The rings in Theorem $n$ are precisely the prime left Goldie orders in $M_{n}(\Delta)$.

These two theorems suggest the following definition of the (row) rank of a subring $R$ of $M_{n}(\Delta)$ [5]. For $R \subseteq M_{n}(\Delta)$ and $0 \leq k \leq n$, we define (row) rank $R \geq k$ if there exist matrix units $\left\{e_{i j} \mid 1 \leq i, j \leq n\right\}$ for $M_{n}(\Delta)$ and a left order $D$ in $\Gamma=\left\{s \in M_{n}(\Delta) \mid s e_{i j}=e_{i j} s, 1 \leq i, j \leq n\right\}$ such that

$$
R \supseteq \sum_{\substack{i=1, \ldots, k \\
j=1, \ldots, n}} D e_{i j} \cong\left(\begin{array}{ccc}
D & \ldots & D \\
\vdots & & \vdots \\
D & \ldots & D \\
0 & \ldots & 0 \\
\vdots & & \vdots \\
0 & \ldots & 0
\end{array}\right)
$$

Of course we write $\operatorname{rank} R=k$ if $\operatorname{rank} R \geq k$ but $\operatorname{rank} R \nsupseteq k+1$. In this terminology, conditions (1.ii) and (n.ii) state, respectively, that rank $R \geq 1$ and $\operatorname{rank} R=n$. Observe also that the rank of $R$ is defined relative to the specific (inclusion) embedding of $R$ in $M_{n}(\Delta)$. Later we will see that $\operatorname{rank} R$ is essentially independent of the choice of the embedding of $R$ as a large subring of a matrix ring over a division ring (Theorem 2.1).

We let $\Delta^{(n)}$ denote the right $\Delta$-vector space of column $n$-tuples over $\Delta . \Delta^{(n)}$ is a left $M_{n}(\Delta)$-module under matrix multiplication, and so is, by restriction, a left $R$-module. By the rank of an element $r \in R$, written rank $r$, we mean as usual the dimension of $r \Delta^{(n)}$ as a right $\Delta$-vector space.

In view of Theorem 1 and Theorem $n$, it was conjectured in [5] that for $R \subseteq$ $M_{n}(\Delta)$ and $1 \leq k \leq n, \operatorname{rank} R \geq k$ would be equivalent to the following condition.

( $k . \mathrm{i})$ Given any $0 \neq t \in M_{n}(\Delta)$ there exists $r \in R$ with rank $r \geq k$ and with $0 \neq r t \in R$.

This conjecture is, however, readily seen to be false. Let $\Delta$ be any division ring and set $R=\left\{\left(a_{i j}\right) \in M_{3}(\Delta) \mid a_{21}=a_{31}=0\right\}$. Then a direct calculation (Example 1.5) establishes that for each $0 \neq t \in M_{3}(\Delta)$ there exists $r \in R$ with rank $r \geq 2$ and with $0 \neq r t \in R$. On the other hand, as seems intuitively obvious, rank $R=1$.

Fortunately, the situation can be remedied by strengthening the requirement of condition $(k . \mathrm{i})$. The principal achievement of this article is to establish the following result.

THEOREM $k$. The following conditions are equivalent for a subring $R$ of $M_{n}(\Delta)$, $\Delta$ a division ring, $n \geq 2$.

$\left(k . \mathrm{i}^{*}\right)$ Given $t_{1} \ldots, t_{n-1} \in M_{n}(\Delta)$, not all zero, there exists $r \in R$ with $\operatorname{rank} r \geq k$ and with $r t_{1}, \ldots, r t_{n-1} \in R$, not all zero.

(k.ii) $\operatorname{rank} R \geq k$.

Theorem 1 and Theorem $n$ are almost immediate consequences of Theorem $k$ in the special cases $k=1$ and $k=n$. In order to establish Theorem $k$ it will be 
necessary to examine carefully the structure of $\Delta^{(n)}$ as a left $R$-module. The most important techniques in the proof were introduced in [4].

In a sequel to this article we will discuss the relationship between the row rank of $R$ and the dually defined column rank of $R$.

1. The main theorem. Throughout this article, all rings are associative but need not pcssess identity elements. For the basic ring theory we need, we rely on [1] as a reference. Theorem $k$ is established by the equivalence of (i) and (ii) in the following theorem.

THEOREM 1.1. For $R$ a subring of $M_{n}(\Delta), \Delta$ a division ring, $n \geq 2$, and $k$ an integer between 1 and $n$, the following conditions are equivalent.

(i) Given $t_{1}, \ldots, t_{n-1} \in M_{n}(\Delta)$, not all zero, there exists $r \in R$ with $\operatorname{rank} r \geq k$ and with $r t_{1}, \ldots, r t_{n-1} \in R$, not all zero.

(ii) $\operatorname{rank} R \geq k$.

(iii) $\Delta^{(n)}$ is a nonsingular indecomposable ( quasi-) injective left $R$-module with $\operatorname{End}_{R} \Delta^{(n)} \cong \Delta$ acting via right multiplications and $\operatorname{dim} U_{\Delta} \geq k$ for every nonzero $R$ - $\Delta$-subbimodule $U$ of $\Delta^{(n)}$.

(iv) $M_{n}(\Delta)$ is the maximal left quotient ring of $R$ and every essential left ideal of $R$ contains an element of rank $\geq k$.

Before turning to the proof of this theorem, we show how to deduce Theorem 1 and Theorem $n$ as special cases. The restriction $n \geq 2$ is a technicality imposed only by the statement of condition (i).

COROLlaRY 1.2. For $R$ a subring of $M_{n}(\Delta), \Delta$ a division ring, the following conditions are equivalent.

(i) Given $0 \neq t \in M_{n}(\Delta)$ there exists $r \in R$ with $0 \neq r t \in R$; i.e., $M_{n}(\Delta)$ is the maximal left quotient ring of $R$.

(ii) $\operatorname{rank} R \geq 1$.

PROOF OF COROLlaRY 1.2. (ii) $\Rightarrow$ (i) follows from the corresponding part of Theorem 1.1. If (i) holds, then, as is easily verified, $R$ is a nonsingular large subring of $M_{n}(\Delta)$. Hence, given any finite sequence $t_{1}, \ldots, t_{m} \in M_{n}(\Delta)$, with say $t_{1} \neq 0, I=\left\{r \in R \mid r t_{i} \in R, i=1, \ldots, m\right\}$ is an essential left ideal of $R$. Then $I t_{1} \neq 0$ so that there exists $r \in R$ with $r t_{1} \neq 0$ and $r t_{i} \in R$ for each $i=1, \ldots, m$. This establishes condition (i) of Theorem 1.1 with $k=1$, and therefore (i) $\Rightarrow$ (ii) in the corollary.

COROLlARY 1.3. For $R$ a subring of $M_{n}(\Delta), \Delta$ a division ring, the following conditions are equivalent.

(i) Given any $t \in M_{n}(\Delta)$ there exists $r \in R$ with $r$ invertible in $M_{n}(\Delta)$ and $r t \in R$; i.e., $M_{n}(\Delta)$ is the classical left quotient ring of $R$.

(ii) $\operatorname{rank} R=n$.

ProOF OF COROLlaRY 1.3. Again (ii) $\Rightarrow$ (i) follows from the corresponding part of Theorem 1.1. If we assume (i) of this corollary, then $M_{n}(\Delta)$ is the classical left quotient ring of $R$, and so Theorem 1.1(i) with $k=n$ follows from a standard fact about common denominators. Hence rank $R=n$, completing the proof of this corollary. 
It is also worthwhile noting that a change of matrix units may be necessary before one can detect the rank of a subring. For example, if $R \subseteq M_{2}(\mathbf{Q})$ is defined by $R=\left\{\left(\begin{array}{ll}a & b \\ c & d\end{array}\right) \mid a, b, c, d \in \mathbf{Z}, a+c=b+d\right\}$, then $R$ does not contain $\left(\begin{array}{ll}D & D \\ 0 & 0\end{array}\right)=$ $\left\{\left(\begin{array}{l}a b \\ 0\end{array} 0\right) \mid a, b \in D\right\}$ for any subdomain $D$ of the rational numbers. If, however, we replace the standard matrix units

$$
\left(\begin{array}{ll}
1 & 0 \\
0 & 0
\end{array}\right), \quad\left(\begin{array}{ll}
0 & 1 \\
0 & 0
\end{array}\right), \quad\left(\begin{array}{ll}
0 & 0 \\
1 & 0
\end{array}\right), \quad\left(\begin{array}{ll}
0 & 0 \\
0 & 1
\end{array}\right)
$$

by the matrix units

$$
e_{11}=\left(\begin{array}{rr}
0 & -1 \\
0 & 1
\end{array}\right), \quad e_{12}=\left(\begin{array}{rr}
-1 & -1 \\
1 & 1
\end{array}\right), \quad e_{21}=\left(\begin{array}{ll}
0 & 1 \\
0 & 0
\end{array}\right), \quad e_{22}=\left(\begin{array}{ll}
1 & 1 \\
0 & 0
\end{array}\right),
$$

then $M_{2}(\mathbf{Q})=\sum_{i, j=1}^{2} \Gamma e_{i j}$, where $\Gamma=\left\{\left(\begin{array}{cc}a & 0 \\ 0 & a\end{array}\right) \mid a \in \mathbf{Q}\right\} \cong \mathbf{Q}$ and $R=D e_{11}+D e_{12}+$ $D e_{22} \supseteq D e_{11}+D e_{12}$, where $D=\left\{\left(\begin{array}{ll}a & 0 \\ 0 & a\end{array}\right) \mid a \in \mathbf{Z}\right\} \cong \mathbf{Z}$. Hence rank $R \geq 1$ and, in fact, $\operatorname{rank} R=1$ (see Corollary $1.4(\mathrm{~b})$ ).

PROOF OF THEOREM 1.1. We will show the cyclic string of implications (i) $\Rightarrow$ (iii) $\Rightarrow$ (ii) $\Rightarrow$ (i), and also that (iii) $\Leftrightarrow$ (iv). We will frequently quote facts obtained in [4].

(i) $\Rightarrow$ (iii) Condition (i) trivially implies condition (1.i) from which one can readily deduce that $V=\Delta^{(n)}$ is a nonsingular indecomposable injective left $R$ module with End $\operatorname{En}_{R} \cong \Delta$ acting via right multiplications [4, Proposition].

Now suppose that $U$ is a nonzero $R$ - $\Delta$-subbimodule of $V$. Let $0 \neq v \in U$ be arbitrary and complete $v$ to a basis $v, v_{1}, v_{2}, \ldots, v_{n-1}$ for $V$ over $\Delta$. Choose elements $t_{1}, \ldots, t_{n-1} \in M_{n}(\Delta)$ satisfying $t_{i} v=v_{i}$ for $i=1, \ldots, n-1$. Then apply condition (i) to choose $r \in R$ with $\operatorname{rank} r \geq k$ and with $r t_{i} \in R$ for each $i=1, \ldots, n-1$. Now $r v \in U$ and $r v_{i}=r\left(t_{i} v\right)=\left(r t_{i}\right) v \in U$ for each $i=1, \ldots, n-1$. Hence

$$
\operatorname{dim} U_{\Delta} \geq \operatorname{dim}\left(r v \Delta+r v_{1} \Delta+\cdots+r v_{n-1} \Delta\right)=\operatorname{dim} r V=\operatorname{rank} r \geq k .
$$

(iii) $\Rightarrow$ (ii) This is proved by an argument given in [4]. We will sketch the basic outline and refer the reader to pp. 346-348 of [4] for detailed proofs of the assertions below.

Let $v_{1}, \ldots, v_{n}$ be any $\Delta$-basis for $\Delta^{(n)}$ and set $A_{i}=\left\{r \in R \mid r v_{j}=0\right.$ for each $j=1, \ldots, n, j \neq i\}, M=\bigcap_{i=1}^{r_{i}} A_{i} v_{i}, F=\{\delta \in \Delta \mid M \delta \subseteq M\}$. Then $M$ is a nonzero compressible left $R$-module (i.e., $M$ embeds in every nonzero $R$-submodule of itself) and $F$ is a left order in $\Delta$.

By hypothesis, $h=\operatorname{dim} M \Delta_{\Delta} \geq k$, so we may choose $m_{1}, \ldots, m_{h} \in M$ linearly independent over $\Delta$. Complete $m_{1}, \ldots, m_{h}$ to a $\Delta$-basis $m_{1}, \ldots, m_{h}, \ldots, m_{n}$ for $\Delta^{(n)}$. Set $N=\bigcap_{i=1}^{n} C_{i} m_{i}$, where $C_{i}=\left\{r \in R \mid r m_{j}=0\right.$ for each $j=1, \ldots, n$, $j \neq i\}$ and put $E=\{\delta \in \Delta \mid M \delta \subseteq N\}$. Then $E$ is also a left order in $\Delta$.

Next, define $e_{i j} \in M_{n}(\Delta)$ by the requirements $e_{i j} m_{l}=\delta_{j l} m_{i}$ (Kronecker delta) for every choice of $1 \leq i, j, l \leq n$. Then $\left\{e_{i j} \mid 1 \leq i, j \leq n\right\}$ is a set of matrix units for $M_{n}(\Delta)$ and for each $\alpha \in \Delta$ there exists a unique element $a=a(\alpha) \in \Gamma=\{t \in$ $M_{n}(\Delta) \mid t e_{i j}=e_{i j} t$ for all $\left.1 \leq i, j \leq n\right\}$ satisfying $m_{i} \alpha=a m_{i}$ for each $1 \leq i \leq n$. Finally, $D=\{a(\alpha) \mid \alpha \in E\}$ is a left order in $\Gamma$ and $R \supseteq \sum_{1 \leq i \leq h ; 1 \leq j \leq n} D e_{i j}$. Hence $\operatorname{rank} R \geq h \geq k$. (Observe, for later use, that we have actually shown that $\operatorname{rank} R \geq \operatorname{dim} M \Delta_{\Delta}$.) 
(ii) $\Rightarrow$ (i) We will show a bit more than necessary. Let $t_{1}, \ldots, t_{m} \in M_{n}(\Delta)$ be any finite sequence with $t_{1} \neq 0$; we claim that there then exists $r \in R$ with rank $r \geq k, r t_{1} \neq 0$, and $r t_{i} \in R$ for all $i=1, \ldots, m$.

We are given that $M_{n}(\Delta)=\sum_{i, j=1}^{n} \Gamma e_{i j}$ and $R \supseteq \sum_{1 \leq i \leq k ; 1 \leq j \leq n} D e_{i j}$, where $D$ is a left order in $\Gamma=\left\{t \in M_{n}(\Delta) \mid t e_{i j}=e_{i j} t\right.$ for each $\left.1 \leq i, j \leq n\right\}$. Hence we may write each $t_{h}=\sum_{i, j=1}^{n} a^{-1} b_{h i j} e_{i j}$ with $a, b_{h i j} \in D$ for $1 \leq i, j \leq n$ and $1 \leq h \leq m$. Also $b_{1 i_{0} j_{0}} \neq 0$ for some choice of $1 \leq i_{0}, j_{0} \leq n$ because $t_{1} \neq 0$. Define $r \in R$ by

$$
r= \begin{cases}a\left(e_{11}+\cdots+e_{k-1, k-1}+e_{k k}\right) & \text { if } i_{0} \leq k, \\ a\left(e_{11}+\cdots+e_{k-1, k-1}+e_{k i_{0}}\right) & \text { if } i_{0}>k .\end{cases}
$$

It is then routine to check that for each $h=1, \ldots, m, r t_{h} \in \sum_{1 \leq i \leq k ; 1 \leq j \leq n} D e_{i j} \subseteq$ $R$, that $r t_{1} \neq 0$, and that $\operatorname{rank} r=\operatorname{dim} r \Delta_{\Delta}^{(n)}=k$. We omit the details.

(iii) $\Rightarrow$ (iv) Since we have already shown that (iii) $\Leftrightarrow$ (i) it is evident that $M_{n}(\Delta)$ is the maximal left quotient ring of $R$. It remains to show that if $L$ is an essential left ideal of $R$, then $L$ contains an element of rank $\geq k$.

Choose a $\Delta$-basis $v_{1}, \ldots, v_{n}$ for $\Delta^{(n)}$ and set $\bar{A}_{i}=\left\{r \in R \mid r v_{j}=0\right.$ for $j=$ $1, \ldots, n, j \neq i\} . A_{i} \cong A_{i} v_{i}$ under the correspondence $a \leftrightarrow a v_{i}$ for $a \in A_{i}$ because $\Delta^{(n)}$ is a faithful left $R$-module. Since $L$ is an essential left ideal of $R$ and $\Delta^{(n)}$ is a uniform left $R$-module, $\bigcap_{i=1}^{n}\left(L \cap A_{i}\right) v_{i} \neq 0$. By hypothesis there exist elements $u_{1}, \ldots, u_{k} \in \bigcap_{i=1}^{n}\left(L \cap A_{i}\right) v_{i}$, linearly independent over $\Delta$. For each $j=1, \ldots, k$ we may write $u_{j}=a_{j} v_{j}$ with $a_{j} \in L \cap A_{j}$. Set $a=\sum_{j=1}^{k} a_{j} \in L$. Since $u_{j}=a v_{j}$ it is clear that rank $a \geq k$, and so $a$ is the desired element of $L$.

(iv) $\Rightarrow$ (iii) Clearly (iv) implies (1.i) and so, as in the proof of (i) $\Rightarrow$ (iii), we can conclude that $\Delta^{(n)}$ is a nonsingular indecomposable injective left $R$-module with $\operatorname{End}_{R} \Delta^{(n)} \cong \Delta$ acting via right multiplications.

Now let $U$ be a nonzero $R$ - $\Delta$-subbimodule of $\Delta^{(n)}$ and fix a $\Delta$-basis $v_{1}, \ldots, v_{n}$ for $\Delta^{(n)}$. Then, because $\Delta^{(n)}$ is a uniform $R$-module, $L=\left\{r \in R \mid r v_{i} \in U\right.$, $1 \leq i \leq n\}$ is an essential left ideal of $R$. By hypothesis, there exists $r \in L$ wth $\operatorname{rank} r=\operatorname{dim} r \Delta_{\Delta}^{(n)} \geq k$. Since $r \Delta^{(n)}=\sum_{i=1}^{n} r v_{i} \Delta \subseteq U \Delta \subseteq U, \operatorname{dim} U_{\Delta} \geq k$.

With this, the proof is completed.

In the course of proving Theorem 1.1 we have obtained some additional information which we now elaborate on and display.

COROLLARY 1.4. Suppose that $R$ is a subring of $M_{n}(\Delta)$ with $\operatorname{rank} R=k \geq 1$. Then the following are true.

(a) Given any elements $t_{1}, \ldots, t_{m}$ of $M_{n}(\Delta)$ with $t_{1} \neq 0$ there exists $r \in R$ with rank $r \geq k, r t_{1} \neq 0$ and $r t_{i} \in R$ for each $i=1, \ldots, m$.

(b) For any $\Delta$-basis $v_{1}, \ldots, v_{n}$ of $\Delta^{(n)}, \bigcap_{i=1}^{n} A_{i} v_{i}$ is a nonzero compressible left $R$-module and $\operatorname{rank} R=\operatorname{dim}\left(\bigcap_{i=1}^{n} A_{i} v_{i}\right) \Delta_{\Delta}$, where $A_{i}=\left\{r \in R \mid r v_{j}=0\right.$ for $1 \leq j \leq n, j \neq i\}$.

(c) $\Delta^{(n)}$ contains a unique minimal $R-\Delta$-subbimodule $U$ with $\operatorname{dim} U_{\Delta}=\operatorname{rank} R$.

ProOF. (a) was proven in showing that (ii) $\Rightarrow$ (i) in Theorem 1.1, and the first assertion in (b) was noted in (iii) $\Rightarrow$ (ii). It is also clear from Theorem 1.1 that when $\operatorname{rank} R \geq 1, \operatorname{rank} R=\min \left\{\operatorname{dim} U_{\Delta} \mid U\right.$ is a nonzero $R$ - $\Delta$-subbimodule of $\left.\Delta^{(n)}\right\}$. Hence the proof of this corollary can be completed by showing that given any $\Delta$-basis $v_{1}, \ldots, v_{n}$ of $\Delta^{(n)},\left(\bigcap_{i=1}^{n} A_{i} v_{i}\right) \Delta$ is the unique minimal nonzero $R$ - $\Delta$-subbimodule of $\Delta^{(n)}$, where $A_{i}=\left\{r \in R \mid r v_{j}=0\right.$ for $\left.1 \leq j \leq n, j \neq i\right\}$. 
To see this, set $M=\bigcap_{i=1}^{n} A_{i} v_{i}$. If now $W$ is any nonzero $R$ - $\Delta$-subbimodule of $\Delta^{(n)}$ then $M \cap W \neq 0$ because $\Delta^{(n)}$ is uniform. Since $M$ is compressible there exists an $R$-monomorphism $\alpha: M \rightarrow M \cap W$, and, because $\Delta^{(n)}$ is quasi-injective, $\alpha$ extends to an element of $\operatorname{End}_{R} \Delta^{(n)} \cong \Delta$ which we also denote by $\alpha$. Hence $M \alpha \subseteq M \cap W \subseteq W$ and therefore $M \Delta=M \alpha \Delta \subseteq W \Delta \subseteq W$, which proves that $M \Delta$ is the unique minimal $R$ - $\Delta$-subbimodule of $\Delta^{(n)}$. From part (b) we know that $\operatorname{dim} M \Delta_{\Delta}=\operatorname{rank} R$.

EXAMPLE 1.5. Let $\Delta$ be a field and set $R=\left\{\left(a_{i j}\right) \in M_{3}(\Delta) \mid a_{21}=a_{31}=0\right\}$, a subring of $M_{3}(\Delta)$. We verify here the two assertions made in the Introduction.

(a) For each $0 \neq t \in M_{3}(\Delta)$ there exists $r \in R$ with $\operatorname{rank} r \geq 2$ and with $0 \neq r t \in R$; and

(b) $\operatorname{rank} R=1$.

For (a), let $0 \neq t=\left(a_{i j}\right) \in M_{3}(\Delta)$ be given. If $a_{21}=a_{31}=0$, then $t \in R$ and we may choose $r$ to be the identity matrix. If $a_{21} a_{31} \neq 0$, then choose

$$
r=\left(\begin{array}{ccc}
0 & 0 & 1 \\
0 & a_{31} & -a_{21} \\
0 & 0 & 0
\end{array}\right) .
$$

If $a_{21} \neq 0$ and $a_{31}=0$, then choose

$$
r=\left(\begin{array}{lll}
0 & 1 & 0 \\
0 & 0 & 1 \\
0 & 0 & 0
\end{array}\right) ;
$$

similarly, if $a_{21}=0$ and $a_{31} \neq 0$ take

$$
r=\left(\begin{array}{lll}
0 & 0 & 1 \\
0 & 1 & 0 \\
0 & 0 & 0
\end{array}\right) .
$$

In every case, rank $r \geq 2$ and $0 \neq r t \in R$.

For (b), we apply Corollary 1.4(b) with

$$
v_{1}=\left(\begin{array}{l}
1 \\
0 \\
0
\end{array}\right), \quad v_{2}=\left(\begin{array}{l}
0 \\
1 \\
0
\end{array}\right), \quad v_{3}=\left(\begin{array}{l}
0 \\
0 \\
1
\end{array}\right)
$$

Then

$$
\bigcap_{i=1}^{3} A_{i} v_{i}=\left(\begin{array}{l}
\Delta \\
0 \\
0
\end{array}\right)
$$

and so rank $r=\operatorname{dim}\left(\bigcap_{i=1}^{3} A_{i} v_{i}\right) \Delta_{\Delta}=1$.

2. Independence of rank. The definition of rank $R$ that we used in $\S 1$ was dependent on the (inclusion) embedding of $R$ in $M_{n}(\Delta)$. The purpose of this section is to show that, when it is $\geq 1$, the rank of $R$ is fundamentally independent of the choice of the embedding of $R$ in a matrix ring over a division ring. We begin by slightly extending the definition of the rank of $R$.

Let $\phi: R \rightarrow M_{n}(\Delta), \Delta$ a division ring, be a ring monomorphism such that, regarding $\phi(R)$ as a subring of $M_{n}(\Delta), \operatorname{rank} \phi(R)=k$. Then we write $\operatorname{rank}_{\phi} R=k$. 
THEOREM 2.1 (Independence of rank)。 Let $\phi: R \rightarrow M_{n}(\Delta)$ and $\psi: R \rightarrow$ $M_{m}(\Gamma)$ be ring monomorphisms with $\Delta$ and $\Gamma$ division rings. If $\operatorname{rank}_{\phi} R \geq 1$ and $\operatorname{rank}_{\psi} R \geq 1$, then $n=m, \Delta \cong \Gamma$, and $\operatorname{rank}_{\phi} R=\operatorname{rank}_{\psi} R$.

It should be evident that the case when $\operatorname{rank}_{\phi} R=0$ or $\operatorname{rank}_{\psi} R=0$ must be excluded from consideration. For example, if $\phi: \Delta \rightarrow M_{1}(\Delta)=\Delta$ is the identity map and $\psi: \Delta \rightarrow M_{2}(\Delta)$ is defined by $\psi(x)=\left(\begin{array}{ll}x & 0 \\ 0 & x\end{array}\right)$, then $\operatorname{rank}_{\phi} \Delta=1$ while $\operatorname{rank}_{\psi} \Delta=0$.

From part (iii) of Theorem 1.1 and Corollary 1.4(c) we know that if $\operatorname{rank}_{\phi} R \geq$ 1 , then (with respect to the $R$-module structure naturally induced by $\phi: R \rightarrow$ $\left.M_{n}(\Delta)\right) \Delta^{(n)}$ is a faithful nonsingular indecomposable injective left $R$-module with End $_{R} \Delta^{(n)} \cong \Delta$ (acting as right multiplications) and with $\operatorname{rank}_{\phi} R=\operatorname{dim} U_{\Delta}$, where $U$ is the unique minimal $R-\Delta$-subbimodule of $\Delta^{(n)}$. A similar statement is also true for $\Gamma^{(m)}$ as a left $R$-module. Hence the preceding theorem is established by the following standard proposition. We include a proof for the sake of completeness.

PROPOSITION 2.2. If $V$ is a faithful nonsingular indecomposable injective left $R$-module and $W$ is any nonsingular indecomposable injective left $R$-module, then $W \cong V$.

Proof. Choose $0 \neq w \in W$. Since $W$ is nonsingular and indecomposable there exists a uniform left ideal $I$ of $R$ with $I w \cong I$ under the $R$-isomorphism $\phi$ defined by $\phi(r w)=r$ for $r \in I$. Since $V$ is faithful there exists $v \in V$ with $I v \neq 0$; then $\psi(r)=r v$ for $r \in I$ defines an $R$-isomorphism from $I$ onto $I v$ because $I$ is uniform and $V$ is nonsingular. Since $V$ is injective and $W$ is indecomposable, $\phi \psi$ extends to an $R$-monomorphism $\theta$ of $W$ into $V . \theta$ is the desired isomorphism because $W$ is injective and $V$ is indecomposable.

\section{REFERENCES}

1. C. Faith, Lectures on injective modules and quotient rings, Lecture Notes in Math., vol. 49, Springer-Verlag, Berlin, 1967.

2. C. Faith and Y. Utumi, On noetherian prime rings, Trans. Amer. Math. Soc. 114 (1965), 53-60.

3. N. Jacobson, Structure of rings, Amer. Math. Soc. Colloq. Publ., vol. 37, Amer. Math. Soc., Providence, R.I., 1964.

4. J. M. Zelmanowitz, Large rings of matrices contain full rows, J. Algebra 73 (1981), 344-349.

5. __ On the rank of subrings of matrix rings, Proc. 4th Internat. Conf. on Representations of Algebras (V. Dlab, Ed.), Carleton Univ., Ottawa, 1984.

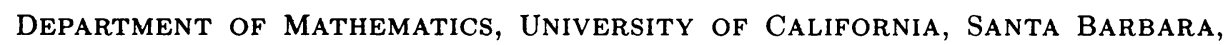
CALIFORNIA 93106 